\title{
Réflexion actuelle sur la prévision hydrométéorologique
}

\author{
Thinking over hydrometeorological forecasting
}

par D. Duband

SHF

For the alert, hydrological and hydrometeorological flow rates forecasting for either all the flood hydrogram or a part of it should result from probality graduating of observed / foreseen measurements uncertainties and modelling coupled uncertainties. Forecasting the rapid flood flow rates from observed and foreseen rain falls and the slow flood flow rates and water height from upstream-downstream water propagation, leans on the transfer delais, each one specific to a catchment area. It has to be frequently updated, following Meteo France meteorological forecasting.

Acquisition, transfer and data processing, rapid, reliable, flexible communications and experts in hydrology and meterology should be available.

\section{IINTRODUCTION}

La prévision des crues rapides dans les bassins versants supérieurs de rivières et fleuves ainsi que les bassins d'affluents intermédiaires, principalement en régions de haute et de moyenne montagne de quelques centaines à quelques milliers de kilomètres carrés, où la transformation de la pluie en débit est prépondérante par rapport au processus de propagation d'un hydrogramme de crue déjà constitué, nécessite une imbrication étroite de la météorologie dynamique et de l'hydrologie, concrétisée par l'hydrométéorologie opérationnelle, permettant ainsi d'augmenter le délai d'anticipation (fig. 1) $[11,12]$.

Cette démarche qui n'a pas encore totalement acquis ses lettres de noblesse en France a été initiée à EDF-DTG dans les années 60-70, en créant une culture météorologique chez les hydrologues par intégration d'ingénieurs de la météorologie.

Il faut avoir une approche moderne de la prévision opérationnelle qui concilie techniques sophistiquées et compétences humaines, tout en se gardant d'une fuite en avant, avec comme atouts indispensables:

- Un réseau fiable et pérenne de stations de mesure pluviométriquesnivométriques-thermométriques-hydrométriques avec acquisition en temps réel des données, complété par des mesures de radar pluviométrique.

- Des modèles hydrologiques globaux (pluie-débit, propagation) parci- monieux, robustes et expérimentés réalisant à chaque pas de temps la transformation de la pluie brute observée et prévue en variations futures de débits de crue, parfois complétée par une propagation hydraulique de crue, associés à une fourchette d'incertitudes, fréquemment réactualisés dans leurs projections futures.

- Des prévisions météorologiques atmosphériques, à différentes échéances (moyen terme, court terme, immédiates) ainsi que l'observation de l'état de l'atmosphère chaque demi-heure ou heure (mosaïque radars, satellites géostationnaires, radars portés...).

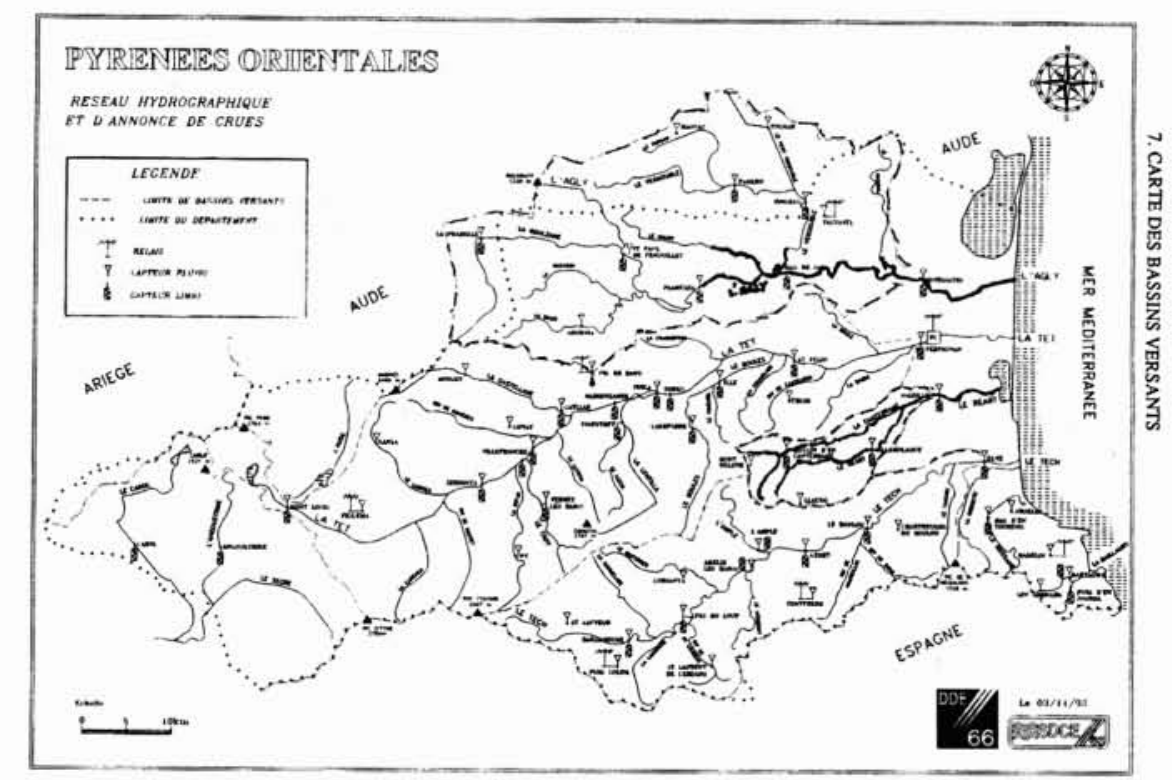



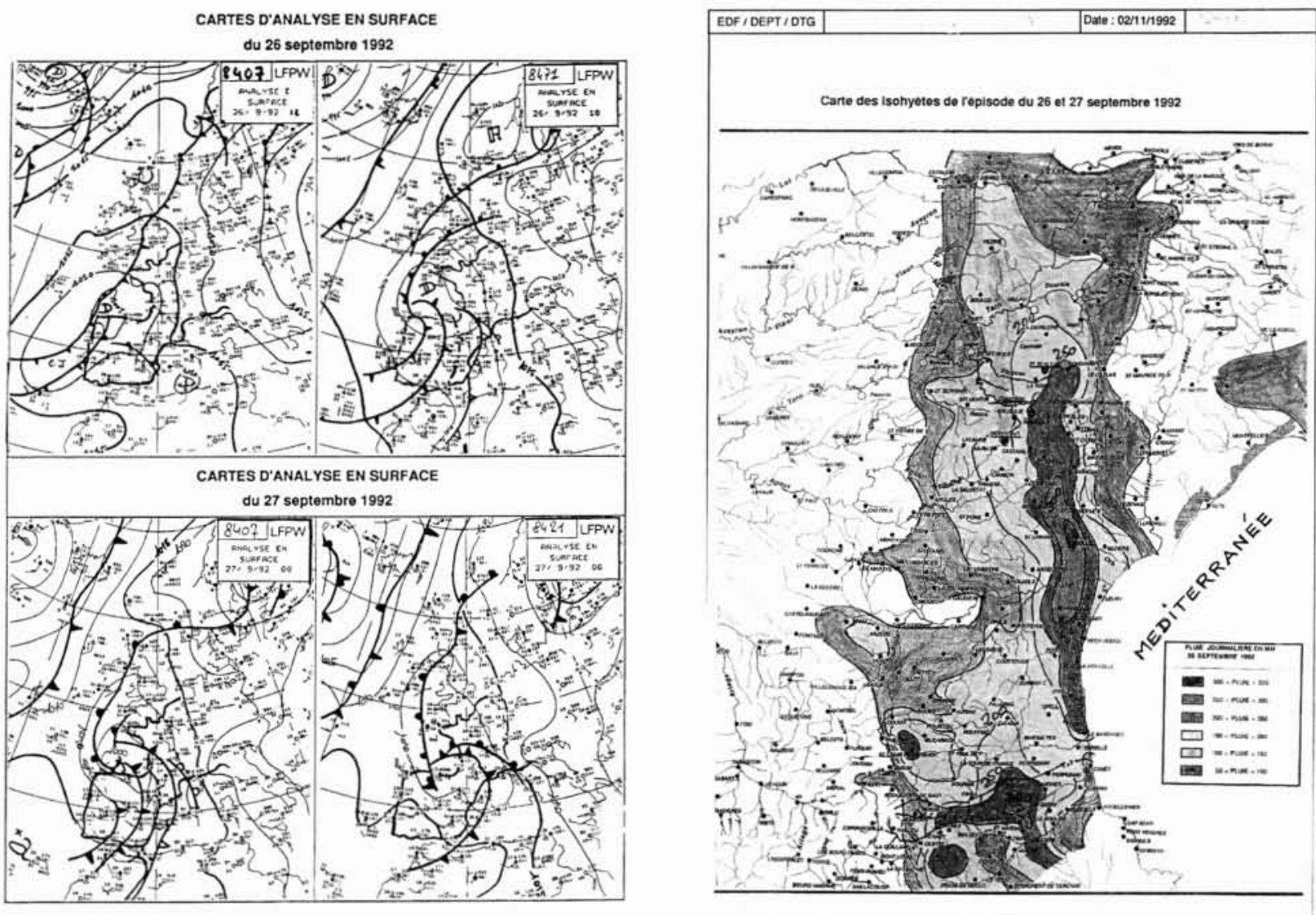

CRUE DU DOURDOU A BEDOS $658 \mathrm{~km}^{2}$
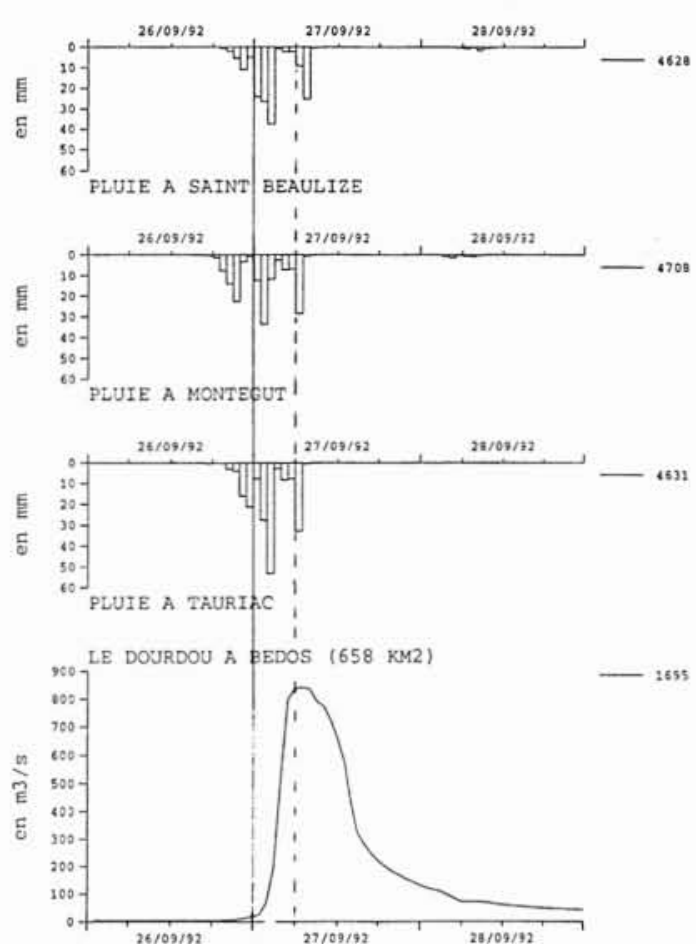

1. Outils de l'hydrométéorologie opérationnelle.

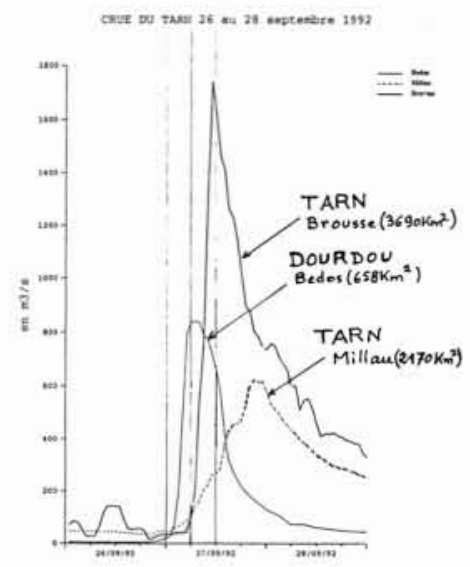

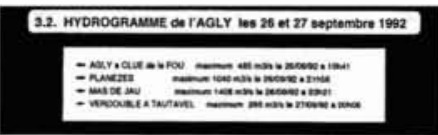

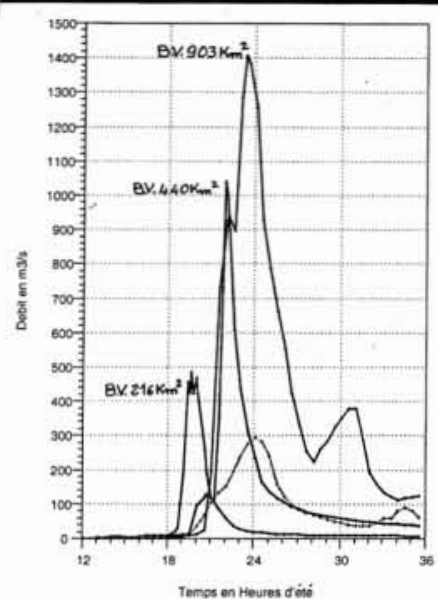

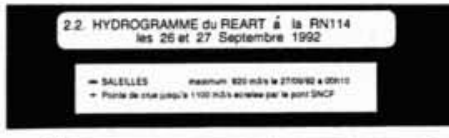

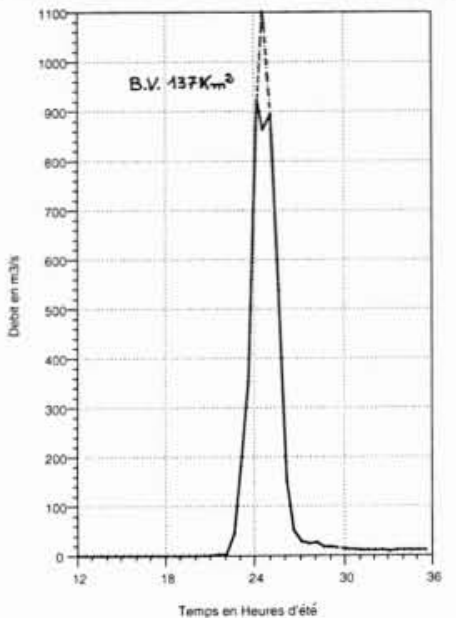


- Des prévisions numériques de précipitations, avec incertitude.

- Des prévisionnistes hydrométéorologues experts, capables d'interpréter et d'adapter les résultats de modèles de prévision météorologiques aux calculs et scénarios des modèles hydrologiques.

- Un dialogue permanent en alerte et en crise entre hydrologues et météorologues.

- Un retour d'expérience organisé, par un contrôle et une analyse systématique des écarts prévision-réalisation.

Alors toute cette infrastructure locale et régionale, dont les bassins versants constituent l'ossature, peut contribuer à mieux cerner l'évolution des crues par l'observation spatiotemporelle de l'état actuel-récent-antérieur et par l'anticipation prévue des phénomènes (précipitation, débit), et fournir efficacement une aide à la décision des gestionnaires de crise.

A la relecture des actes du colloque SHF de novembre 1987 [1] sur la prévision des crues, il est frappant de constater qu'au cours de ces dix dernières années les réseaux de mesure en temps réel se sont nettement améliorés et étendus, mais que les progrès en prévision hydrologique opérationnelle sont restés très modestes.

Bien que le chemin des crues prévues soit pavé de bonnes intentions : logiciels sophistiqués-simulation séduisantes, aujourd'hui il y a encore très peu de systèmes opérationnels de prévision présentant des caractères de rigueur qui permettraient d'envisager un transfert d'expérience à d'autres cas de façon satisfaisante selon la remarque faite alors par P.A. Roche en introduction à ces journées. Certes devant les difficultés multiples et dans un contexte d'information physique qui sera toujours incomplète, insuffisante, affectée d'incertitudes de mesure, il convient d'être modeste et prudent, mais au moins ne pourrait-on pas conduire en partenariats quelques expériences de terrain pendant 5 à 10 ans avec un véritable plan d'expérience? Exemple des projets: Ardèche (MÉTÉOFRANCE/EDF-DTG/LTHE) et Garonne (DIREN PYR./M.F/U. Toulouse)

\section{DES RÉSEAUX DE MESURE EN TEMPS RÉEL (LA TÉLÉSUR- VEILLANCE)}

Il ne s'agit pas de faire un bilan des systèmes existants et en projets, qui ont progressé depuis 10 ans, mais de souligner quelques aspects importants.

Le suivi à pas de temps fin (l'heure ou la demi-heure, par exemple) des intensités ponctuelles de la pluie au sol, de la température de l'air, du niveau d'eau et du débit correspondant dans une section de rivière, représente une étape essentielle et un progrès considérable.[10]

On peut ainsi obtenir à chaque pas de temps présent une "photographie» (simultanéité) des événements hydrométéorologiques sur un ou plusieurs bassins versants, ainsi que le "film» de l'évolution récente, surtout lors de variations rapides, et cela permet déjà d'esquisser une prévision immédiate pour les débits. Le coût d'investissement en matériels et les coûts de maintenance conduisent à sélectionner judicieusement les stations de mesure témoins parmi les réseaux existants (ou à créer), en particulier pour bien représenter la spatialisation des pluies.

Pour assurer la fiabilité du système temps réel, l'effort a porté sur la qualité :

- Des capteurs, pluviomètres enregistreurs des intensités et cumuls, sondes thermorésistives de température, capteurs de niveau adaptés au régime de la rivière avec courbe de tarage régulièrement étalonnée ; en favorisant l'utilisation de capteurs intelligents avec auto-diagnostic de fonctionnement et signalisation de seuil d'alerte.
- Système d'acquisition soit avec mémorisation locale, soit avec mémorisation sur un concentrateur regroupant plusieurs stations, complété par une interrogation régionale; le projet de station PLQ 2000 de la DE/MATE permettra d'améliorer la communication avec des utilisateurs externes.

- Pour des raisons de sécurité, un certain nombre de sites sont équipés du double système de transmission radio-satellite géostationnaire ou téléphone-satellite géostationnaire, avec des capteurs doublés et indépendants; la protection contre la foudre a été considérablement améliorée ; [13].

- Le traitement informatique, avec la miniaturisation des ordinateurs et l'augmentation de leur capacité mémoire, ainsi que la possibilité de travailler en réseaux, les visualisations chronologiques et spatiales sur écrans, apporte une souplesse et une rapidité d'interprétation très précieuses dans l'aide à la prévision et le contrôle des données, et il permet de «rafraîchir» régulièrement les prévisions.

\section{III —LA MODÉLISATION OPÉRATION- NELLE}

Deux processus sont à considérer :

- La transformation de la pluie brute observée en débit de ruissellement direct, lorsque la relation pluie-débit est prépondérante dans le bassin.

- La transformation d'une crue amont en crue aval dans une rivière ou fleuve lorsque la propagation hydraulique est prépondérante (en traitant de préférence les débits pour ensuite revenir au niveau d'eau, dans les sections de référence).

On développera ici le premier processus, le second étant mieux modélisé et appréhendé en prévision opérationnelle, mais susceptible d'améliorations.

\subsection{Relation Pluie-Débit pour la prévision}

Pour une utilisation opérationnelle en prévision, on a besoin d'une modélisation spécifique fort différente des besoins de l'hydrologie appliquée à la compréhension et à la reconstitution du cycle de l'eau: simuler n'est pas prévoir. Cependant des améliorations de la connaissance peuvent contribuer à l'un ou l'autre des objectifs.

Les mécanismes spatio-temporels précis de l'infiltration et du ruissellement direct sont d'une part en relation avec la distribution chronologique et spatiale des intensités de pluie mesurées ponctuellement, améliorables spatialement par le traitement des images radar, mais plus ou moins proche de la pluie réelle (inconnue) reçue par le bassin versant, et, d'autre part dépendent de l'état estimé des réserves hydriques souterraines et de l'état d'humidification des sols pour une morphologie-pédologie-géologie-couverture végétale particulières au bassin versant, qui fonctionne comme un ou plusieurs filtres retardataires en réponse aux impulsions de la pluie.

En présence d'informations incomplètes, avec incertitudes tant physiques que métrologiques, il faut une modélisation simple, robuste, fiable, parcimonieuse, s'appuyant sur un processus physique globalisé, ce qui pose le choix des échelles d'espace et de temps structurantes.

Dans la modélisation opérationnelle deux approches principales sont proposées :

- La modélisation en "continu" qui consiste à reproduire à pas de temps fin (horaire ou plurihoraire) la chronique des débits à l'exutoire d'un bassin versant (crues, tarissement, étiages) d'après un système à 4 ou 5 réservoirs en série et parallèle avec leurs lois de remplissage et de vidange ayant comme données d'entrée la précipitation et la température de l'air, exemples : les modèles GR3-GR4 du Cemagref, le 
modèle Coriandre d'EDF-DTG (qui stimule aussi le manteau neigeux); l'étalonnage s'effectue d'après 2 ou 3 ans d'observations.

- La modélisation "événementielle» qui consiste à reproduire l'hydrogramme de ruissellement direct, essentiellement dans la montée de crue, la pointe de crue et le début de la décrue jusqu'au tarissement, d'après le hyetogramme d'averse, en s'appuyant sur le processus de transformation de la pluie brute en pluie efficace, et le transfert de celle-ci en débit à l'exutoire du bassin : c'est l'approche dite hydrogramme unitaire et différence première de la fonction de transfert (DPFT) ; elle est étalonnée d'après un échantillon d'averses-crues. [2, 3, 4].

Dans ce qui suit, je ferai plutôt un plaidoyer en faveur de l'approche globale du type hydrogramme unitaire ou fonction de transfert moyenne, étant conscient d'être ainsi partial [5].

Actuellement (et depuis quelques dizaines d'années) on peut considérer que c'est le moins mauvais des modèles opérationnels, le plus simple et le plus pratique à mettre en œuvre et dont on identifie bien les faiblesses. Il me semble par ailleurs que le modèle en continu peut servir à initialiser certains paramètres du modèle événementiel, en particulier pour certaines fonctions de production pluie brute-pluie efficace (état des réserves hydriques, fusion d'un manteau neigeux...), l'évolution du débit de base...

\section{- 3.2 Rappels et commentaires sur la méthode DPFT}

En ayant défini le pas de temps unitaire $[t-1, t]$, qui peut varier de 1 heure à 6 heures pour un bassin versant de 1000 $\mathrm{km}^{2}$ selon sa géomorphologie, on note :

$P B_{1}$ : la pluie brute moyenne sur le versant cumulée entre $t-1$ et $t$ (il s'agit de la pluie de bassin). $P E_{t}$ :

la pluie effective ou efficace cumulée entre $t-1$ et $t$, contribuant au ruissellement direct, le rendement marginal $P E_{t} / P B_{t}$ voisin de 1 contribue à la violence de la crue.

$Q_{t}^{r}: \quad$ représente le débit de ruissellement $Q_{t}=Q_{b}+Q_{t}^{r}: \quad$ représente le débit total, somme du débit de base et du débit de ruissellement direct (le coefficient d'écoulement global de crue est : $\left.0,1<\Sigma P E_{t} / \Sigma P B_{t}<0,7\right)$.

$\Delta Q_{t}=q_{t}=Q_{t}-Q_{t-1}$ : représente la variation de débit de ruissellement direct entre $t-1$ et $t$ (on considère que la variation du débit de base est faible devant celleci, sauf en cas de crue double).

La fonction de production utilisée par EDF-DTG est du type soustractif :

$$
P E_{t}=P B_{t}-D_{\lambda}\left(P B_{t} / P B_{t}+D_{t}\right) \quad[6]
$$

où $D$, représente un déficit d'écoulement inversement proportionnel à l'état de saturation du bassin versant caractérisé par un indice des débits antérieurs $I Q A_{t}=\lambda Q_{t}+(1-\lambda)$ $I Q A_{t-1}$ avec $0 \leq \lambda \leq 1$ (qui peut être simplement le débit de base $Q_{b}$ ), complété éventuellement (en zone méditerranéenne) par un indicateur d'humidification superficielle du sol, d'après les pluies antérieures, $I P B A_{t}=\theta P B_{t}+(1-\theta)$ $I P B A_{t-1}$ avec $0 \leq \theta \leq 1$ où $\theta$ et $\lambda$ caractérisent la mémoire).

$$
D_{t}=\frac{K}{H\left(Q_{t-i} P B_{t-i}\right)} \quad(K \text { est un coefficient saisonnier })
$$

Le calage se fait par ajustement itératif des paramètres d'après les pluies efficaces déconvoluées par la DPFT pour chacun des hydrogrammes de l'échantillon.

Mais il y a d'autres modélisations de la fonction de production : modèle à réservoirs, TOPMODEL, HORTON... ou des combinaisons de ces modèles [7].
La différence première de la fonction de transfert moyenne (DPFT) de la pluie efficace (mm) en débit de ruissellement direct $\left(\mathrm{m}^{3} / \mathrm{s}\right)$ est définie par les $\mathrm{m}$ coefficients :

$$
a_{1}, a_{2}, a_{3}, \ldots a_{m}
$$

ce qui permet d'établir les coefficients de la fonction de transfert moyenne (FT) :

$$
\begin{aligned}
& A_{1}=a_{1}, A_{2}=a_{1}+a_{2}, A=a_{1}+a_{2}+a_{3}, \ldots A_{m}=a_{1}+\ldots+a_{m} \\
& \text { Ainsi } q_{t}=\sum^{m} a_{i} P E_{t-i+1} \quad \text { (DPFT) } \\
& \text { Et } Q_{t}^{r}=\sum \text { AiPE } E_{t-i+1}=\sum^{t} q_{1} \quad \text { (FT) }
\end{aligned}
$$

La FT sert essentiellement à effectuer une prévision de débit en mode dégradé, sans recalage sur le dernier débit observé.

Rappelons que le calcul des coefficients a s'effectue (par convolution-déconvolution) à partir d'un échantillon de 20 à 40 épisodes, choisi avec des pluies sur l'ensemble du bassin et pour des crues au moins quinquennales, avec des mesures de débits de bonne qualité (fig. 2).

La DPFT et la FT représentent une véritable signature de chaque bassin versant, avec le pas de temps unitaire approprié (fig. 3).

Il est certain que chaque bassin versant est composé de sous-bassins structurants $(2,3, \ldots p)$ et que la DPFT et FT moyennes se décomposent ainsi :

$$
a_{i}=\sum_{J=1}^{2 a \dot{a} p} a_{i j} ; A_{i}=\sum_{J=1}^{2 \grave{a} p} A_{i j}
$$

L'identification de ces fonctions de transfert composantes est rarement possible faute de données de mesure de débit sur les sous-bassins. Cependant lorsque c'est le cas, on pourra aussi identifier la fonction de production propre à chaque sous-bassin.

Cela nous rapproche de la notion de pluie distribuée telle que l'on pourrait la comprendre : en cas d'averse très localisée qui ne concerne qu'un sous-bassin, il est évident qu'à la fois la fonction de production globale initialisée par la lame d'eau moyenne sur le bassin versant complet, et sa DPFT/FT seront défaillantes quand il s'agit de produire des variations de débit et des débits corrects à l'exutoire de celui-ci, si l'on ne dispose pas du modèle production/transfert spécifique au sous-bassin concerné.

\subsection{Prévision de variations de débits}

La prévision des débits à l'instant $\mathrm{t}$ pour les échéances $t+1$, $t+2, t+3, \ldots t+h$ connaissant débits et pluies spatiales à $t$, et antérieurement $(t-1, t-2, \ldots t-i)$ se comprend simplement :

$$
\hat{Q}_{t+h}=Q t+\hat{q}_{t+1}+\hat{q}_{t+2}+\hat{q}_{t+3}+\ldots+\hat{q}_{t+h}+\varepsilon_{t+h}
$$

C'est à dire que l'on ajoute les variations prévues au dernier débit connu à l'instant $t$ pour les pas de temps futurs et l'on procédera ainsi lors de la réactualisation à l'instant $t+1$, etc. :

- la variation prévue à $t+1$ :

$\hat{q}_{t+1}=a_{1} \overline{P E}_{t+1}+a_{2} \hat{P} E_{t}+a_{3} P_{i} \hat{E}_{t-1}+\ldots+\varepsilon_{t+1}$

$\hat{P} E_{t-i}$ est la pluie efficace déduite de la pluie brute observée $P B_{t-i}$.

$\overline{P E}_{t+1}$ pluie efficace déduite de la pluie brute prévue $\overline{P B}_{t+1}$

$\varepsilon_{t+1}$ est la somme des incertitudes associées à la prévision ; 


\section{Transformation de pluie}
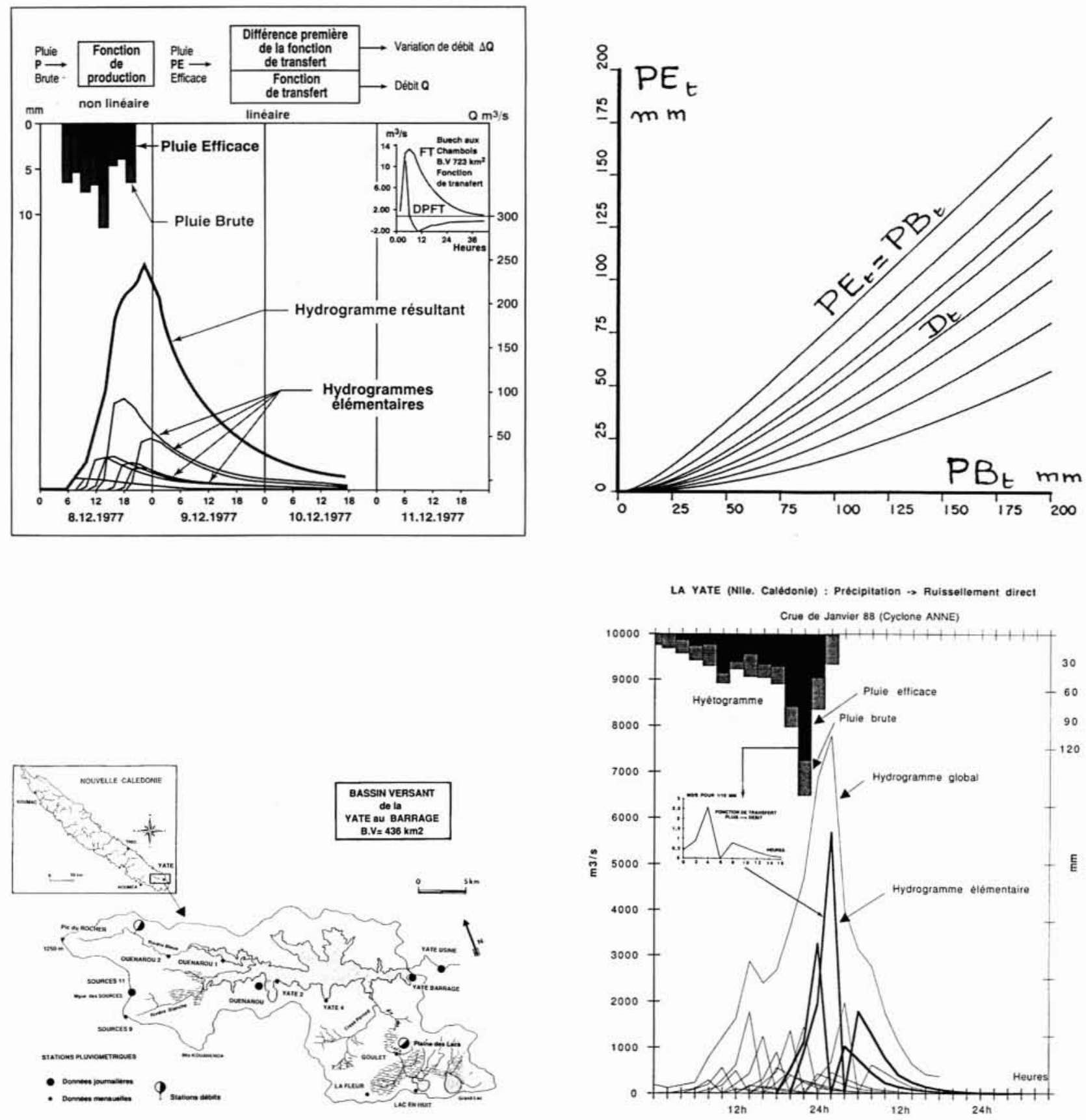

2. Eléments pour le calcul des coefficients a.

- de même la prévision de variation entre $t+1$ et $t+2$ se note :

$\hat{q}_{t+2}=a_{1} \overline{P E}_{t+2}+a_{2} \overline{P E}_{t+1}+a_{3} P \hat{E}_{t}+a_{4} P \hat{E}_{t-1}+\ldots+\varepsilon_{t+2}$

$P E_{t+2}$ et $P E_{t+1}$ sont estimées d'après les pluies prévue $\overline{P B}_{t+1}$, $\overline{P B}_{t+2}$, et $\varepsilon_{t+2}$ représente la somme des incertitudes sur la pluie observée, sur la transformation de $P B$ observée en $P E$ et sur la prévision de $P B$.

Or pour un certain nombre de bassins : $a_{1}=0$, ou $a_{1}=a_{2}=0$ (ou sont des coefficients faibles), ce qui réduit d'autant l'incertitude sur les premières variations de débit prévues, en retardant l'incidence des pluies prévues mais on voit bien aussi l'importance progressive que prennent les pluies futures "prévues» en phase d'accroissement de débit de crue, puis en phase de décrue (en relation avec la durée du pas de temps unitaire). Cette présentation fait apparaître la nécessité de calculer un écart-type d'incertitude associée à chaque prévision de variation future : $\sigma_{\varepsilon}=\left(\sigma_{1}^{2}+\sigma_{2}^{2}+\sigma_{3}^{2}\right)^{1 / 2}$ écartstypes: $\sigma_{1}$ de $P B$ mesuré, $\sigma_{2}$ de $P B$ prévu, $\sigma_{3}$ de modèle. 

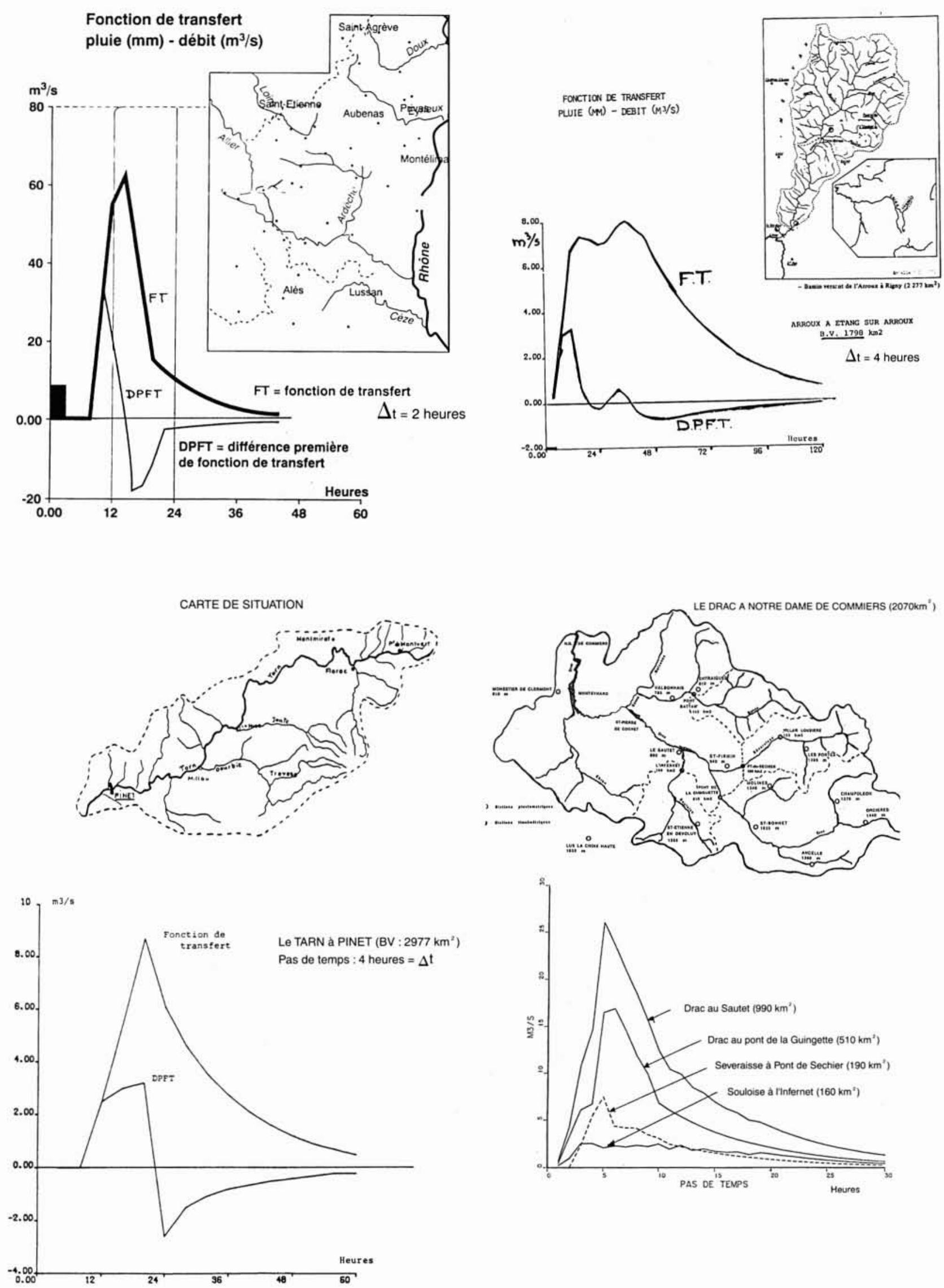

3. Exemples de DPFt et de FT. 


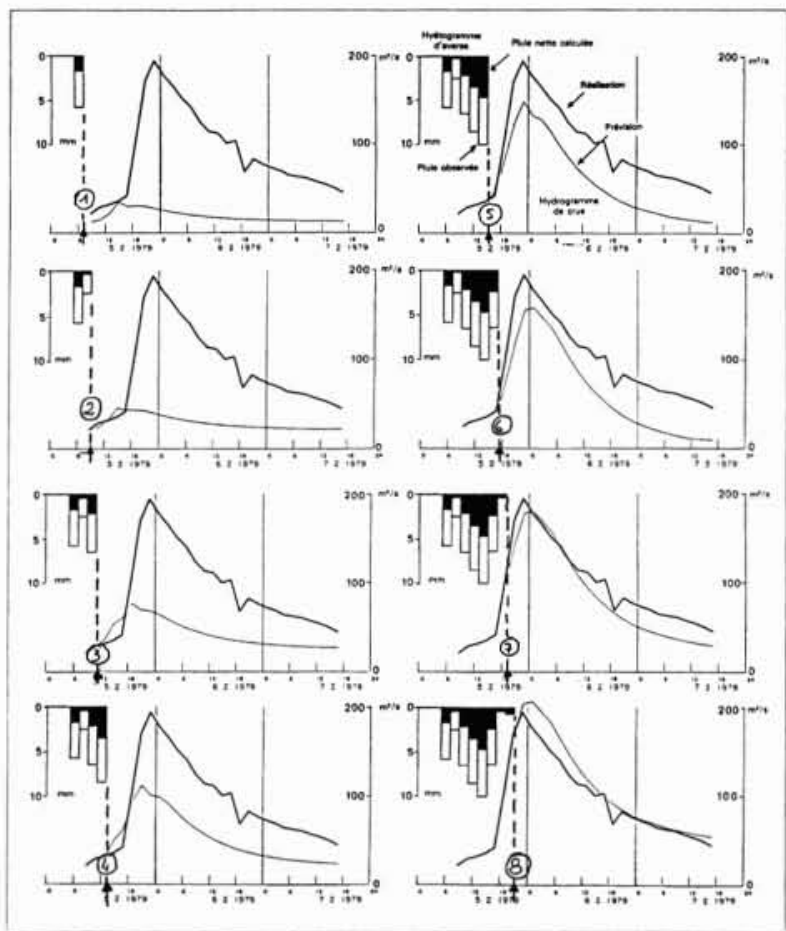

Exemple de prévision de débits de la Bourne à Pont-de-Manne au pas de temps de deux heures, d'après les précipitations observées pendant l'averse des 5 et 6 février 1979

TARN a PINET

PREVISION DE LA CRUE DU BET I NOVEMBAE 1982 AU PAS

DE TEMPS UNITAIRE DE 4h, SANS RECALAGE EN COUAS DE CAUE

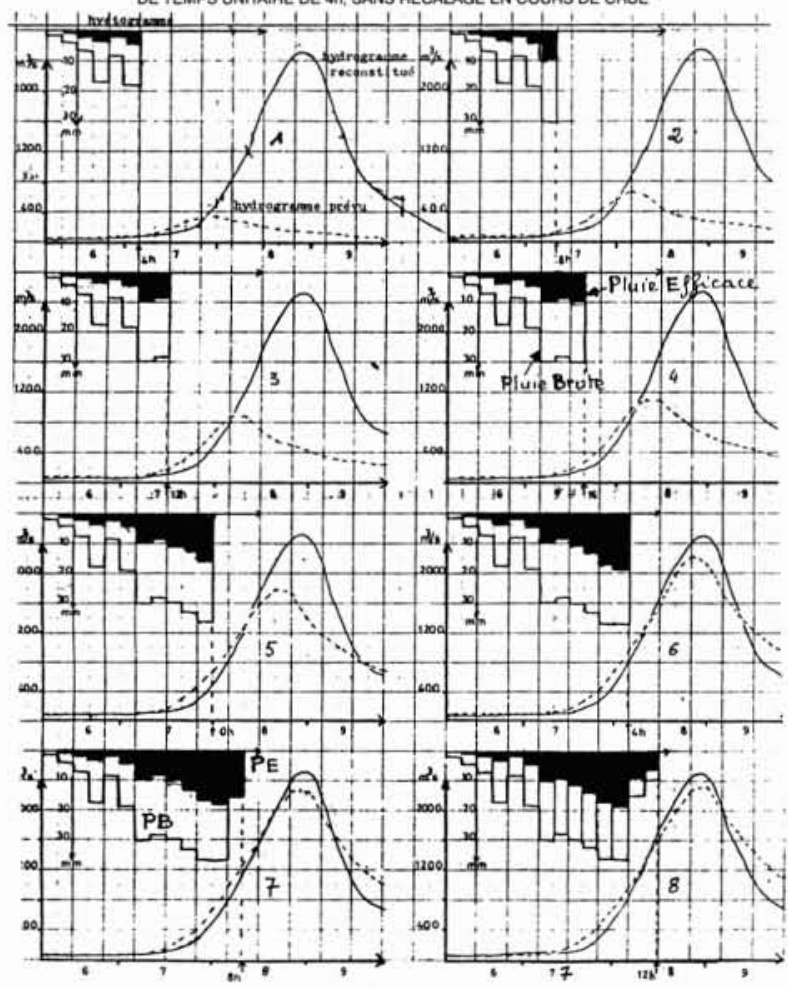

4. Exemples de prévisions de débits.
LA BOURNE A PONT DE MANNE (B. V. $797 \mathrm{~km}^{\prime}$ )

Coefficiens de la fonction de transtert et de sa difference premiere au pas unitaire de 2 heures
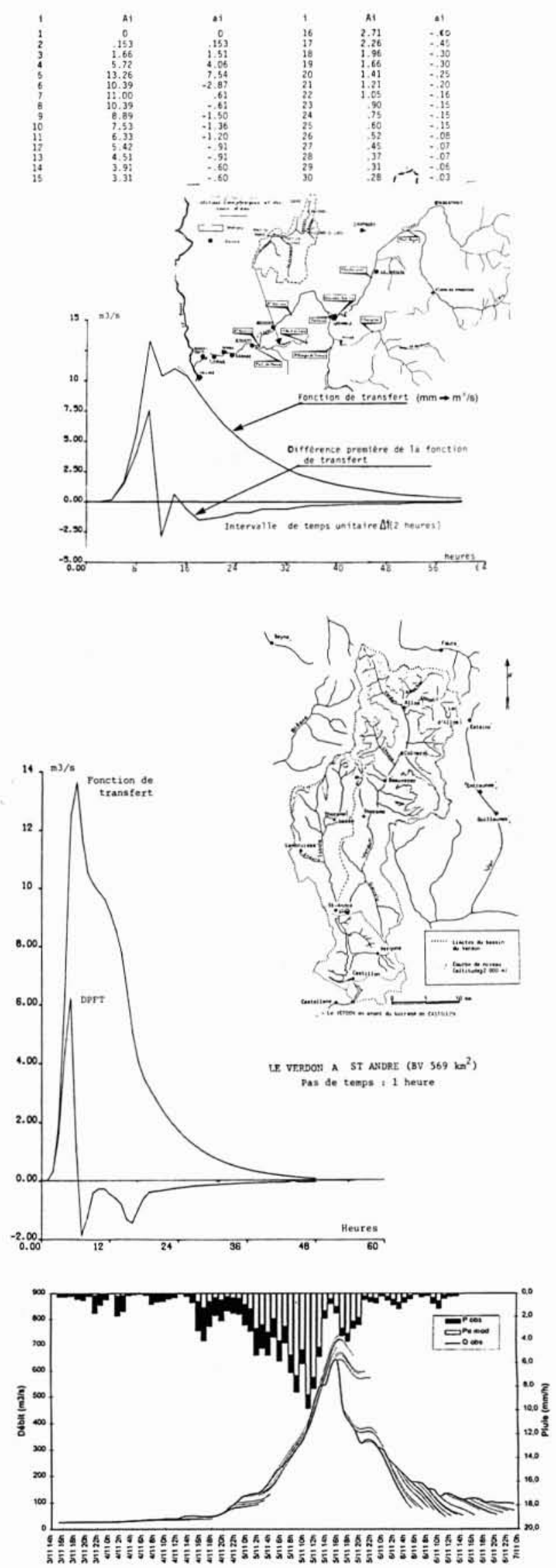
Cette approche de calcul des incertitudes me semble beaucoup plus fondamentale à mettre en œuvre que des procédures multimodèles avec pondérations évolutives, et de recalage "soft" des paramètres d'un modèle qui, bien que séduisantes, me paraissent d'une expérimentation irréaliste pendant une action opérationnelle de prévision de crue rapide par des prévisionnistes aussi chevronnés soient-ils.

\subsection{La prévision des précipitations}

- La première hypothèse, sans doute triviale, mais qui n'est pas sans intérêt, consiste à faire l'hypothèse que les pluies futures seront nulles $\left(\overline{P B}_{t+1}=\overline{P B}_{t+2}=\ldots=0\right)$ ce qui permet de calculer une hypothèse plancher pour les variations prévues de débit futur (fig. 4).

- La seconde hypothèse consiste soit à prendre pour $P B_{t^{+}}$ une valeur égale à $P B_{t}$, ou soit prendre les valeurs fournies par ASPIC (Modélisation MÉTÉO France).

- Une troisième hypothèse proposée par le professeur OBLED consiste à utiliser la valeur moyenne de distribution conditionnelle des pluies journalières par bassin-versant fournie par la méthode de sélections de situations météorologiques analogues à la situation prévue pour le jour $\mathrm{j}$, puis celle prévue pour le jour $j+1$, puis de désagréger celle-ci au pas de temps unitaire approprié à chaque bassin pour estimer un scénario de hyetogramme d'averse.

- Une quatrième hypothèse consiste à utiliser les prévisions numériques de précipitation à pas de temps de 6 heures établies par MF, qui sont en net progrès pour les épisodes frontaux d'hivers, mais conservent des incertitudes importantes pour les épisodes convectifs en termes de localisation et intensité.

- Enfin une approche prometteuse pourrait résulter du programme MAP (Mesoscale Alpine Project) pendant l'automne 1999, en application du modèle de recherche MesoNH de MÉTÉO-FRANCE, qui devrait ouvrir la voie à une prévision numérique des pluies orographiques de meilleure qualité (échelles $10 \mathrm{~km}, 1$ heure) selon les espoirs de P. Bougeault)[9].

\section{IV —OBSERVATIONS ET PRÉVISIONS MÉTÉOROLOGIQUES}

Les sorties numériques journalières du modèle européen (CEPMMT) qui est traité à Readings permettent d'établir une prévision en tendance des jours $j+3$ à $j+7$. A partir des valeurs prévues de pression et température sur l'Europe de l'Ouest, EDF utilise un modèle statistique de recherche de situations météorologiques analogues associées aux précipitations locales sur 33 bassins versants témoins, dans un fichier quotidien de 1953 à 1998 [8].

Des jours $j$ à $j+2$, le modèle ARPEGE mis au point par MÉTÉO-FRANCE prend le relais. Ce modèle est lancé par le Service Exploitation de MÉTÉO-FRANCE deux fois par jour à $00 \mathrm{~h}$ et à $12 \mathrm{~h}$ UTC avec un pas de temps de 6 heures : il permet de prévoir plus finement les risques météorologiques et soit en confirme progressivement la sévérité et la localisation régionale, soit en atténue le caractère de gravité. Malheureusement la prévision numérique des précipitations reste encore imprécise à court terme malgré une amélioration appréciable de la localisation spatiale des champs précipitants, pour des zones de plusieurs milliers de $\mathrm{km}^{2}$, son utilisation demeure très qualitative.

Le MÉTÉOTEL avec écran de visualisation en temps réel des images radar, satellitaires, est un outil maintenant indispensable au prévisionniste hydrologue, pour le temps réel étendu et l'affinement de la prévision hydrologique immédiate.
Ces visualisations spatiales :

- Images radars, au pas du $1 / 4$ d'heure, simples ou composites du réseau ARAMIS qui permettent de localiser les zones précipitantes et ainsi d'initialiser la prévision de débit sur le ou les bassins concernés; elles fournissent l'information primordiale qu'est l'arrêt des précipitations locales.

- Images du satellite MÉTÉOSAT dans le visible et l'infrarouge, temps réel, toutes les demi-heures ou heures, permet de faire une liaison avec les prévisions de MÉTÉOFRANCE à $6 \mathrm{~h} 00$ et $12 \mathrm{~h} 00$ pour ce qui concerne la position des masses d'air à l'échelle régionale.

- On peut d'ailleurs animer la succession des images passées, effectuer une superposition de plusieurs types d'images.

Enfin, l'image en temps réel de la localisation (à $2 \mathrm{~km}$ ) des impacts de foudre par le système MÉTÉORAGE permet de situer les phénomènes orageux et des phénomènes convectifs et d'en suivre l'évolution.

Mais l'interprétation pertinente de cet ensemble d'informations ne peut se faire que dans le cadre des sorties numériques d'ARPEGE pour les 24 heures en cours avec ainsi la «toile de fond" d'une analyse de données météorologiques prévues.

Ainsi, ces informations soumises à l'expertise de l'hydrométéorologue prévisionniste constituent un complément utile et indispensable aux résultats des mesures temps réel de la dernière collecte automatique et peut aider à effectuer une extrapolation à très court terme des débits de crue prévus.

On ne peut encore les prendre en compte sous forme numérique (assimilation ?) dans les modèles hydrologiques de prévision de débit, mais elles constituent plutôt un appui en termes de scénario qualitatif futur.

Le défi de la prévision des valeurs numériques d'intensité de pluie pour les 6 prochaines heures est vraiment difficile, mais MÉTÉO-FRANCE a déjà entrepris de le relever en s'investissant dans des projets tels que ASPIC et SYMPOSIUM pour les 3 premières heures, et elle a engagé une réflexion pour l'échéance de 3 à 6 heures, qui fait le lien avec le plus proche pas de temps des prévisions météorologiques numériques qui est de 6 heures.

On voit ainsi toute l'utilité des prévisions météorologiques emboîtées dans l'espace et dans le temps de 7 jours jusqu'à l'observation en temps réel de la situation atmosphérique sur l'Europe, avec mises à jour régulières de ces calculs numériques, dans la progressivité tendance-détection, prévision, confirmation du risque-alerte et suivi de l'état atmosphérique.

\section{$\mathrm{V} \square$ CONCLUSION}

Nous avons essayé de montrer tout au long de cette communication la nécessaire et indispensable complémentarité de la

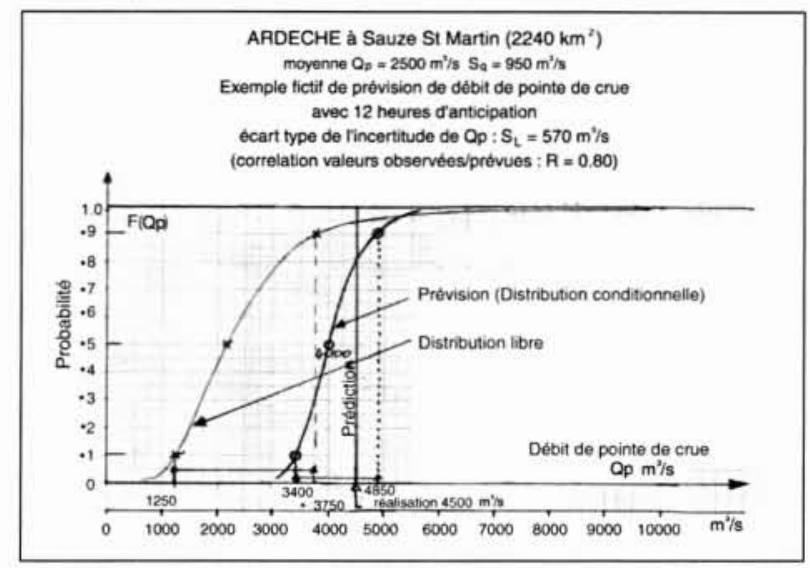

5. Graduation en probabilité des incertitudes. 
météorologie dynamique et l'hydrologie opérationnelle à travers toute une infrastructure qui comporte comme socle de base les réseaux de mesure en temps réel (sol et atmosphère) puis s'articule sur les sorties numériques de modèles de prévisions météorologiques qui permettent de guider les modèles de prévisions de débit de crue à quelques heures (1 à 24 heures) calculées d'après une approche simplifiée du processus pluie-débit dans un bassin versant.

Il faut insister sur la nécessaire recherche d'une graduation en probabilité des incertitudes (notre ignorance) qui affectent la valeur la plus probable calculée pour chaque prévision (fig. 5). Cela implique un archivage organisé des retours d'expérience.

En temps réel, la cohérence et la validation spatio-temporelle des observations et des prévisions sont assurées respectivement par des experts météorologues et hydrologues qui fonctionnent en synergie et en étroite communication lors de situation de crise. Chacun exerce un métier à part entière dans des domaines suffisamment vastes et complexes où il ne peut y avoir d'improvisation, ni de substitution des uns par les autres.

Il nous parait plus efficace de former un hydrométéorologue opérationnel compte tenu de la multiplicité des applications pointues de la météorologie.

Toutefois une meilleure compréhension du processus pluie-débit par les météorologues peut également contribuer à améliorer la précision de la mesure de l'intensité réelle et la répartition du champ de pluie (lame d'eau) reçu par le bassin, ainsi que la prévision des caractéristiques (intensité, localisation, persistance...) du champ de pluie à quelques heures d'échéance qui sont utiles à l'hydrologue.

Enfin si actuellement nous sommes mieux préparés à prévoir et à gérer des crues importantes inférieures à la crue centennale, il n'est pas sûr que nos infrastructures hydrométéorologiques nous permettent de prévoir une crue de type millennal $\left(10^{-3}\right)$, ou des crues de type centennal généralisées à une surface importante du territoire français (type mai 1856).

\section{RÉFÉRENCES}

[1] Prévisions des crues. -- La Houille blanche $n^{\circ} 5 / 6,1988$ (Colloque SHF, 1987).
[2] Guillot P. et Duband D. - Une méthode de transfert pluie-débit par régression multiple. IASH n 129, 1980.

[3] Duband D., Nalbandis, Obled C., Tourasse P., RodriGUEZ J.Y. - Unit Hydrograph revisited, the first differenced transfert function (FDTF) approach. IAHS $n^{\circ} 190,1990$.

[4] Duband D., Obled C., Rodriguez Y. - Unit Hydrograph revisited, an alternate iterative approach to U.H and Effective Precipitation Identification. Journal of Hydrology $\mathrm{n}^{\circ} 150,1993$.

[5] Obled C., rodriguez Y., GM Saulnier G.M. - Une approche opérationnelle pour la modélisation des bassins versants sujets aux crues éclair. SHF, $23^{e}$ journées de l'Hydraulique, Nimes, 1994.

[6] Garcon R. - Modèle global Pluie-Débit pour la prévision et la prédétermination des crues. Crues de la normale à l'Extrême. Colloque SHF, Lyon, mars 1999.

[7] Vidal J.J., Dupouyet J.P., Murillo T. - SOPHIE, système ouvert de prévision hydrologique informatisé avec expertise. SHF, $25^{\mathrm{c}}$ journées de l'hydraulique, Chambéry, 1998.

[8] Guilbaud S., Obled C. - L'approche par analogies en prévision météorologique : idées générales et application à la prévision journalière des précipitations. La Météorologie n 24, décembre 1998.

[9] Bougeault P. - Météorologie Alpine, un programme de recherche pleine ascension. Atmosphériques $\mathrm{n}^{\circ} 1$, décembre 1998, MÉTÉO-FRANCE.

[10] Tourasse P. - Prévision et télésurveillance hydrométéorologique à EDF. La Houille Blanche n’ 2, 1991.

[11] DDE et DDAF des Pyrénées orientales. - Mission Inter Service de l'eau : la crue du 26 septembre 1992 dans les Pyrénées orientales. La plus forte depuis l'Aiguat d'octobre 1940.

[12] PINTE J. - Descriptif hydro-météorologique de la crue des 26 et 27 septembre 1992 dans le haut bassin du Tarn. Note interne EDF-DTG, Toulouse, 1992.

[13] Lallement C., Tourasse P. - Comportement et utilisation opérationnelle d'un réseau hydroclimatologique en période de crise. SHF, $23^{e}$ journées de l'hydraulique, 14-16 septembre 1994, Nìmes. 